\title{
Case Report \\ Successful Use of Ectopic Pelvic Kidney for Living Related Donation Technical Aspects and Literature Review
}

\author{
R. C. Minnee, ${ }^{1}$ H. J. A. N. Kimenai, ${ }^{1}$ J. van de Wetering, ${ }^{2}$ and J. N. M. Ijzermans ${ }^{1}$ \\ ${ }^{1}$ Department of Surgery, Division of HPB \& Transplant Surgery, Erasmus University Medical Center (Erasmus MC), \\ Rotterdam, Netherlands \\ ${ }^{2}$ Department of Nephrology, Erasmus University Medical Center (Erasmus MC), Rotterdam, Netherlands
}

Correspondence should be addressed to R. C. Minnee; rcminnee@hotmail.com

Received 23 March 2017; Accepted 10 May 2017; Published 4 June 2017

Academic Editor: David Conti

Copyright (C) 2017 R. C. Minnee et al. This is an open access article distributed under the Creative Commons Attribution License, which permits unrestricted use, distribution, and reproduction in any medium, provided the original work is properly cited.

Ectopic pelvic kidneys can provide an additional source of organs for transplantation. They are often excluded from donation in living donation programs mainly due to aberrant vascular and urinary anatomies. We present a donor with an ectopic left kidney, who successfully donated his kidney. The use of ectopic pelvic kidney for living kidney transplantation is a highly demanding surgical procedure but after extensive preoperative investigation in high volume centers with surgical expertise in vascular reconstruction and access surgery, ectopic pelvic kidneys should not be a contraindication for donation and should be considered as a viable option.

\section{Introduction}

Live donor kidney transplantation is the optimal method of renal replacement therapy for most patients with end stage renal disease in terms of short- and long-term results [1]. Shortage of available donor kidneys compels us to expand the supply of living donor kidneys. Essentially, the donor kidney must be anatomically and functionally suitable for transplantation, and the donor must be in good medical and psychological health and left with a good renal function from the remaining kidney.

The use of ectopic pelvic kidneys can provide an additional source of organs for transplantation.

Ectopic pelvic kidney is a relatively rare congenital anomaly in which the kidney fails to ascend to its lumbar position during embryonic development. The incidence of pelvic kidneys is reported as 1 in 1000 autopsies; only routine ultrasound screening in children revealed a lower incidence of 1 in $5000[2,3]$. The location of an ectopic kidney can be pelvic, lumbar, abdominal, and thoracic or crossed fused. Ectopic pelvic kidney slightly occurs more on the left side with predominance in male subjects. Due to malrotation, the ectopic pelvic kidney might have a flattened, discoid shape. This incomplete rotation leads to an undeveloped renal pelvis with a variety of congenital anatomies such as a shorter ureter, defective ureteral drainage, and multiple renal arteries and renal veins. Ectopic pelvic kidneys are often excluded from donation in living donation programs mainly due to these aberrant vascular and urinary anatomies resulting in only a few successful case reports [4-15]. We advocate the use of these kidneys for transplantation in high volume transplant centers and present a successful livingunrelated kidney transplantation of an ectopic pelvic kidney; in addition, we present a review of the literature to focus on the technical aspects.

\section{Case Report}

The donor was a 43-year-old man donating a kidney to his wife. In the past, he underwent arthroscopic meniscus repair on his left knee and micro discectomy of a herniated nucleus pulposus. He did not use any medication and reported no allergies. During workup, an ectopic left kidney and a normally placed right kidney were found. Physical examination was normal with a BMI of 25.7. Blood and urine examination revealed no abnormalities. His measured creatinine clearance was $123 \mathrm{~mL} / \mathrm{min}$. The computed tomography (CT) angiogram performed showed 1 artery, 1 vein, and 1 ureter of the left 


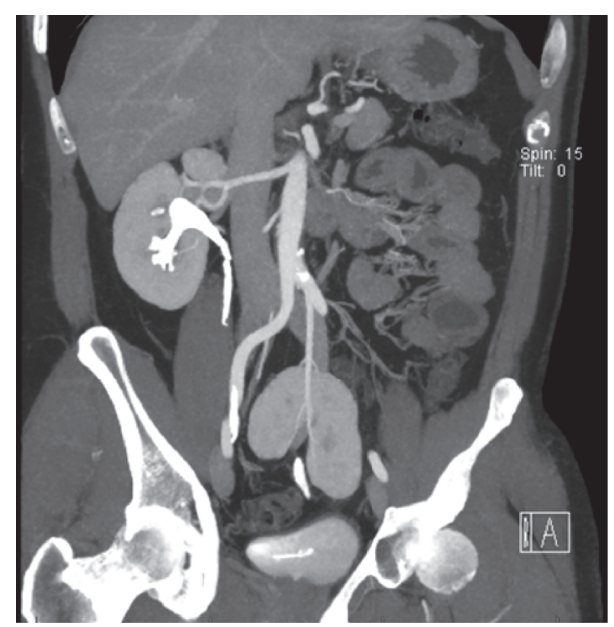

FIGURE 1: CT scan with coronal image of ectopic left pelvic kidney showing the middle polar artery.

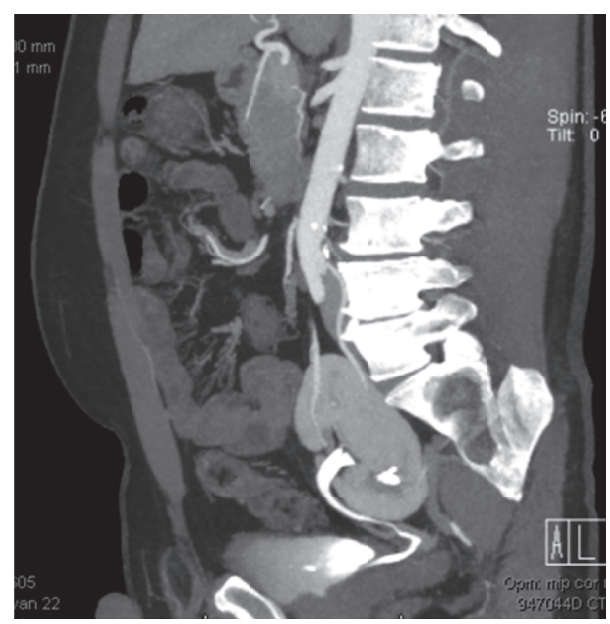

FIGURE 2: CT scan with sagittal image of ectopic left pelvic kidney showing the upper and middle polar artery.

kidney (Figures 1 and 2). The ectopic left kidney had a relative function of $40 \%$ in conventional technetium-99m (Tc-99m) mercaptoacetyltriglycine (MAG3) scan. During the renal transplant multidisciplinary meeting, it was recommended to select the left kidney for donation.

A left open donor nephrectomy was performed through a Gibson incision. The donor kidney had additional blood vessels undetected on CT scan. There were an additional upper polar artery and an additional middle polar artery (Figure 3). On the bench, an end-to-side anastomosis was made between the middle polar artery and the main renal artery with Prolene 7.0 (Figure 4).

The recipient was the donor's 43-year-old wife with end stage renal failure secondary to tubulointerstitial nephritis. The donor kidney was placed in the right iliac fossa. The renal vein was anastomosed to the common iliac vein, whereas the reconstructed renal artery was anastomosed end to side to the external iliac artery and the upper polar artery to the common iliac artery. The anastomosis time for all anastomoses was

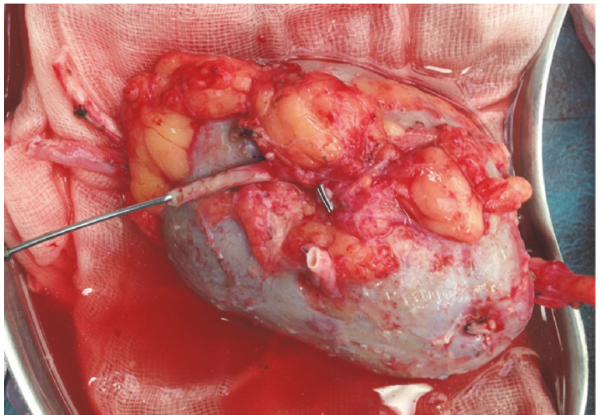

FIGURE 3: Left pelvic kidney with additional upper polar artery and an additional middle polar artery before reconstruction.

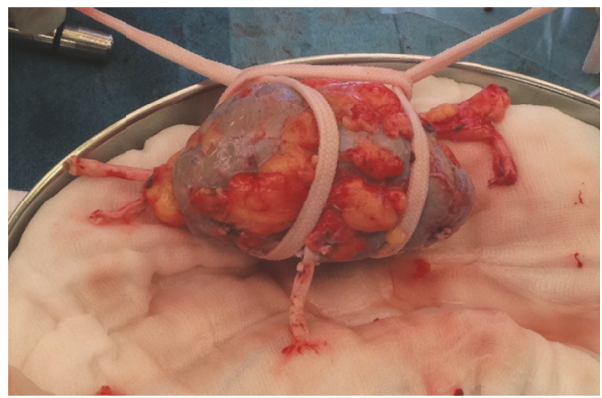

FIGURE 4: After end-to-side reconstruction of the middle polar artery and main renal artery.

32 minutes. The method used to establish urinary continuity was extravesical ureteroneocystostomy and closure of the abdominal wall in layers. The ureteroneocystostomy was stented with an externally draining 8-French catheter for 9 days. According to local protocol, a standard dose of $12.000 \mathrm{U}$ heparin daily was given during the first 5 days due to the arterial reconstruction. The postoperative course of the donor and the patient was uneventful.

\section{Discussion}

The use of an ectopic kidney for transplantation in a living transplant program has been only reported in thirteen patients (Table 1) [4-15]. In all cases, extensive investigation of live donors was carried out. The majority of ectopic kidneys had multiple renal vessels especially renal arteries (77\%). However, despite comprehensive investigation in 4 cases (29\%) (including our case), discrepancy was found between the preoperative imaging and operative findings $[4,11,14]$. In all 4 cases, additional renal veins and/or arteries were visualized. So, even after extensive preoperative imaging, a cautious approach should be undertaken during the donor nephrectomy for the existence of additional vascular structures.

Several studies reported clinicoradiological discrepancies between preoperative CT renal angiography and operative findings ranging from $5 \%$ to $15 \%$ [16-18]. Retrospectively, Johnson et al. could not identify 3 of the 12 discrepancies between CT angiographic and operative findings on image review [16]. This reveals an effect of experience on reporting 


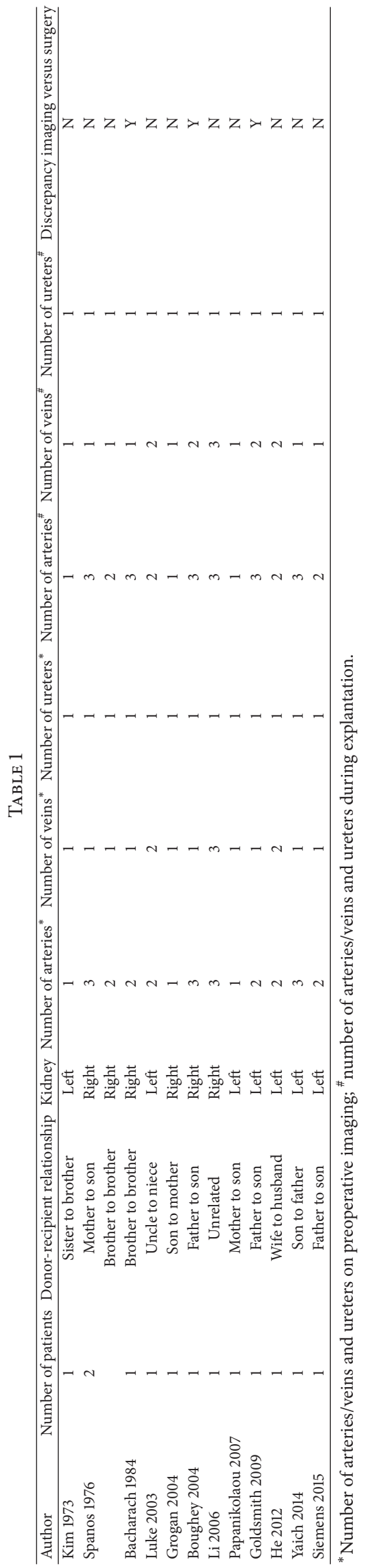




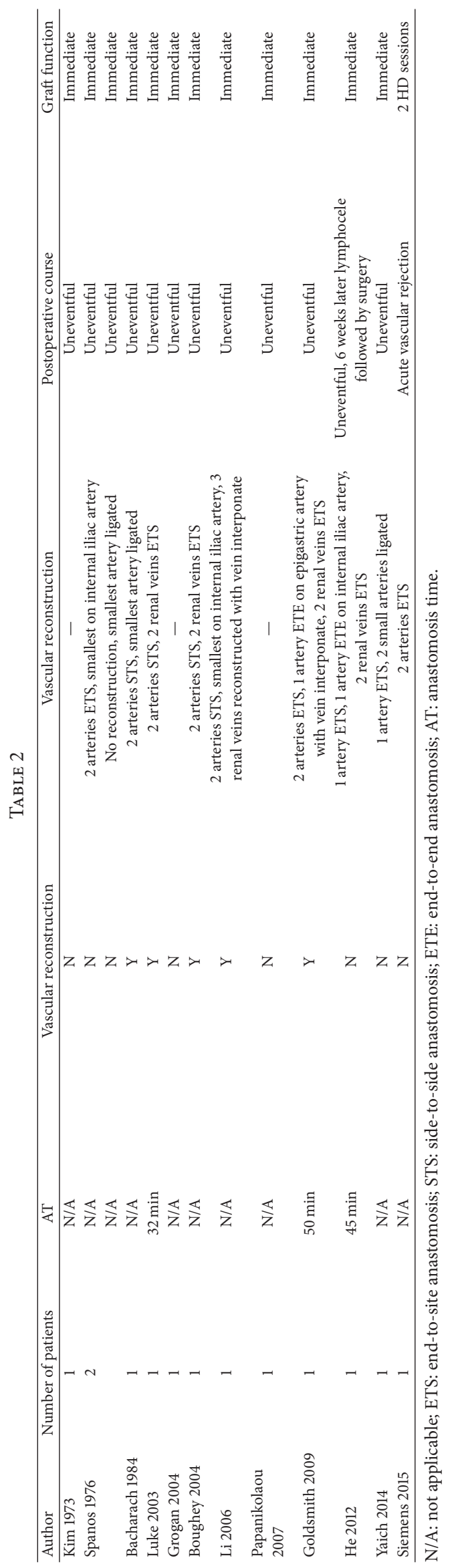


accuracy and greater awareness of potential reporting pitfalls, which was the case in our report.

All kidney grafts showed immediate graft function except in one case requiring two hemodialysis sessions (Table 2). All arterial and venous reconstructions were flawless, showing that reconstruction of multiple renal vessels in ectopic pelvic kidneys is feasible for transplantation [4, 11, 13-15].

Ureteral duplication occurs in approximately $0.8 \%$ of the general population and the incidence of duplicated ureters in ectopic pelvic kidney is unknown [19]. Approximately $50 \%$ of ectopic kidneys have a hydronephrosis. Half of these cases are due to obstruction of the ureteropelvic or the ureterovesical junction ( $70 \%$ and $30 \%$, resp.), 25\% from reflux grade III or greater, and $25 \%$ from the malrotation alone [20]. Despite all aberrant urinary anatomies described in the literature, all transplanted ectopic pelvic kidneys reported in the literature had a normal single ureter and urinary collecting system. When the ureter is very short, ureteroureterostomy or pyelovesicostomy is an alternative option.

Laparoscopic donor nephrectomy has been performed in 2 cases $[8,12]$. Key points for laparoscopic donor nephrectomy are careful interpretation of the preoperative imaging and use of a Endo Retract ${ }^{\mathrm{TM}}$ articulating fan retractor to increase the working space [12].

Hence, these donors should have extensive investigations of the renal vasculature and anatomy variation using computed tomography angiography or magnetic resonance angiography. Intravenous pyelography can also be performed to detect urinary tract abnormality and, additionally, nuclear renography is necessary to assess the renal function of each kidney.

The main limitation in all the case reports is the presence of publication bias. Only successful transplantations are most likely reported and published.

The use of ectopic pelvic kidney for living kidney transplantation is a highly demanding surgical procedure but nonetheless a feasible one. After extensive preoperative investigation in high volume centers with surgical expertise in vascular reconstruction and access surgery, ectopic pelvic kidneys should not be a contraindication for donation and should be considered as a viable option. Since the presence of an ectopic pelvic kidney is a relatively common condition, it must be noted that the use of these kidneys may expand the donor pool in experienced hands.

\section{Conflicts of Interest}

The authors declare that they have no conflicts of interest regarding the publication of this paper.

\section{References}

[1] C. L. Davis and F. L. Delmonico, "Living-donor kidney transplantation: A review of the current practices for the live donor," Journal of the American Society of Nephrology, vol. 16, no. 7, pp. 2098-2110, 2005.

[2] S. P. Dretler, C. Olsson, and R. C. Pfister, "The anatomic, radiologic and clinical characteristics of the pelvic kidney: an analysis of 86 cases," Journal of Urology, vol. 105, no. 5, pp. 623-627, 1971.
[3] C. P. Sheih et al., "Renal abnormalities in schoolchildren," Pediatrics, vol. 84, no. 6, pp. 1086-1090, 1989.

[4] M. D. Bacharach and N. L. Tilney, "Use of an ectopic pelvic donor kidney for transplantation," Transplantation Proceedings, vol. 16, no. 6, pp. 1663-1665, 1984.

[5] E. L. Grogan, W. A. Nylander, and D. Shaffer, "Living-related transplantation of an ectopic pelvic kidney [3]," Transplantation, vol. 77, no. 6, p. 953, 2004.

[6] C. H. Kim and O. Fjeldborg, "Transplantation of pelvic kidney: A case report," Scandinavian Journal of Urology and Nephrology, vol. 7, no. 2-3, pp. 236-238, 1973.

[7] V. Papanikolaou, D. Giakoustidis, N. Antoniadis, N. Ouzounidis, D. Takoudas, and A. Antoniadis, "Living-related transplantation of an ectopic pelvic kidney," International Journal of Urology, vol. 14, no. 4, pp. 357-359, 2007.

[8] T. A. Siemens et al., "Pelvic kidney for living transplantation: case report and review of the literature," Jornal Brasileiro de Nefrologia, vol. 37, no. 3, pp. 418-421, 2015.

[9] P. K. Spanos, R. Weil, R. L. Simmons, and J. S. Najarian, "Successful transplantation of ectopic kidneys from living related donors," The American Journal of Surgery, vol. 131, no. 3, pp. 360 362, 1976.

[10] S. Yaich et al., "Use of a pelvic kidney for living transplantation," Saudi Journal of Kidney Diseases and Transplantation, vol. 25, no. 2, pp. 408-409, 2014.

[11] J. C. Boughey, O. E. Emovon, F. Afzal et al., "Living donor transplantation of a pelvic kidney," Clinical Transplantation, vol. 18, no. 3, pp. 336-338, 2004.

[12] B. He and A. Mitchell, "Laparoscopic donor nephrectomy for ectopic kidney," Transplantation Proceedings, vol. 44, no. 10, pp. 3051-3054, 2012.

[13] J. Li, S. Nadalin, A. Paul et al., "The symptomatic pelvic kidney: A new source of organs for living donor kidney transplantation?” Transplantation, vol. 82, no. 9, pp. 1241-1242, 2006.

[14] P. J. Goldsmith, M. Marco, Z. Hussain, C. Newstead, J. P. A. Lodge, and N. Ahmad, "Kidney Transplantation Using the Inferior Epigastric Vessels for Multiple Anastomoses from a Pelvic Kidney," Journal of the American College of Surgeons, vol. 209, no. 1, p. -e3, 2009.

[15] P. P. W. Luke, V. C. McAlister, A. M. Jevnikar et al., "Use of a pelvic kidney for living transplantation: Case report and review of the literature," American Journal of Transplantation, vol. 3, no. 2, pp. 235-238, 2003.

[16] J. E. Johnson, E. J. Loveday, L. J. Archer, P. Lear, and M. J. Thornton, "Preoperative evaluation of live renal donors using multislice CT angiography," Clinical Radiology, vol. 60, no. 7, pp. 771-777, 2005.

[17] J. F. Platt, J. H. Ellis, M. Korobkin, and K. Reige, "Helical CT evaluation of potential kidney donors: Findings in 154 subjects," American Journal of Roentgenology, vol. 169, no. 5, pp. 13251330, 1997.

[18] J. J. Del Pizzo, G. N. Sklar, J. W. You-Cheong, B. Levin, T. Krebs, and S. C. Jacobs, "Helical computerized tomography arteriography for evaluation of live renal donors undergoing laparoscopic nephrectomy," Journal of Urology, vol. 162, no. 1, pp. 31-34, 1999.

[19] J. T. J. Privett, W. D. Jeans, and J. Roylance, "The incidence and importance of renal duplication," Clinical Radiology, vol. 27, no. 4, pp. 521-530, 1976.

[20] C. J. Shapiro E, "Anomalies of the upper urinary tract," in Campbell-Walsh Urology, pp. 3123-3160, Elsevier, 2011. 


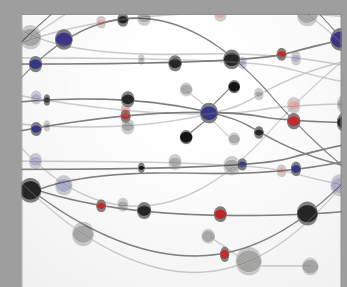

The Scientific World Journal
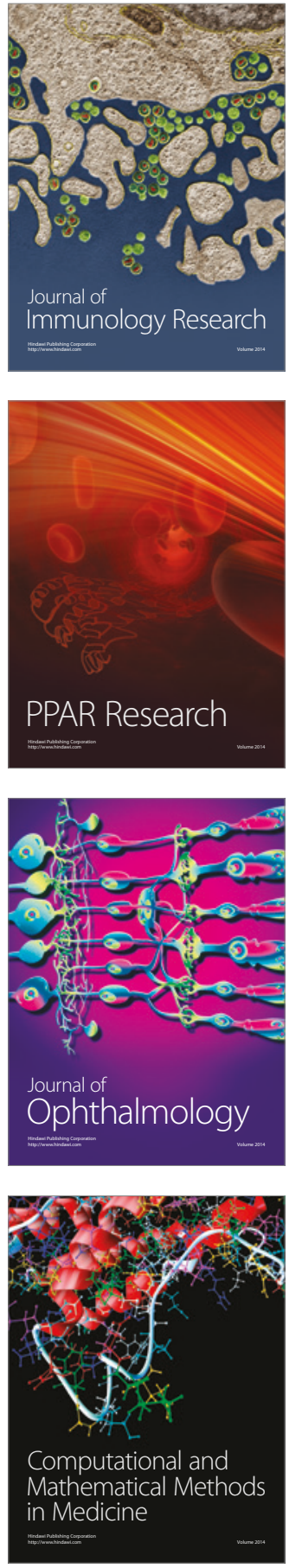

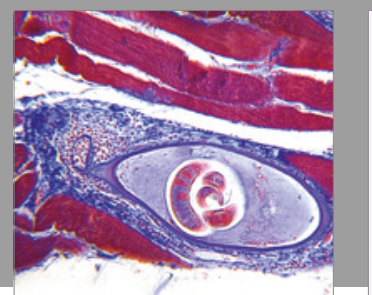

Gastroenterology Research and Practice
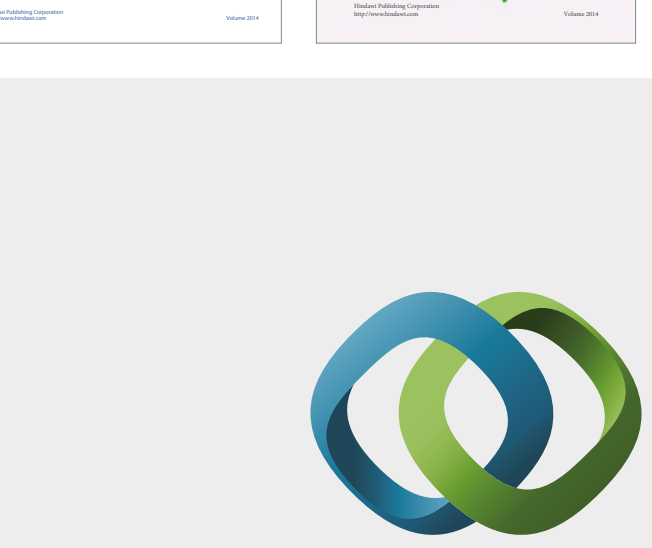

\section{Hindawi}

Submit your manuscripts at

https://www.hindawi.com
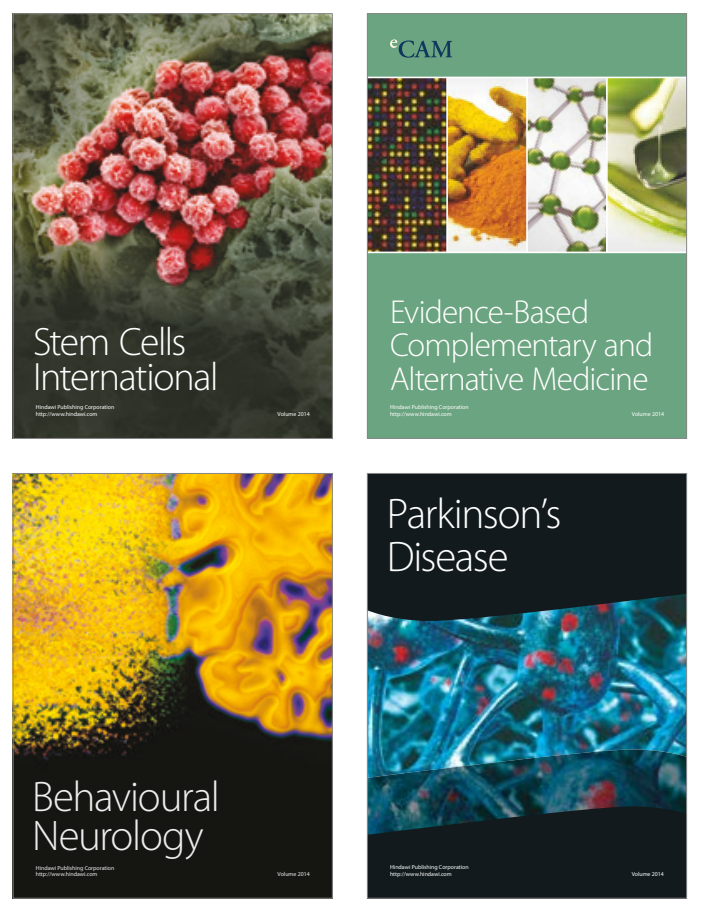
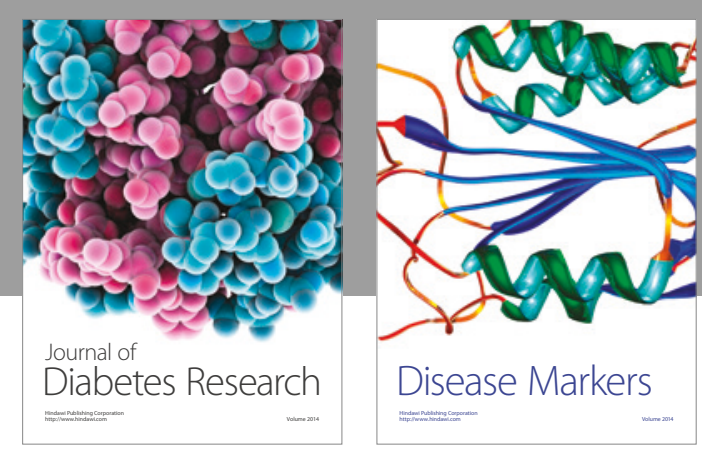

Disease Markers
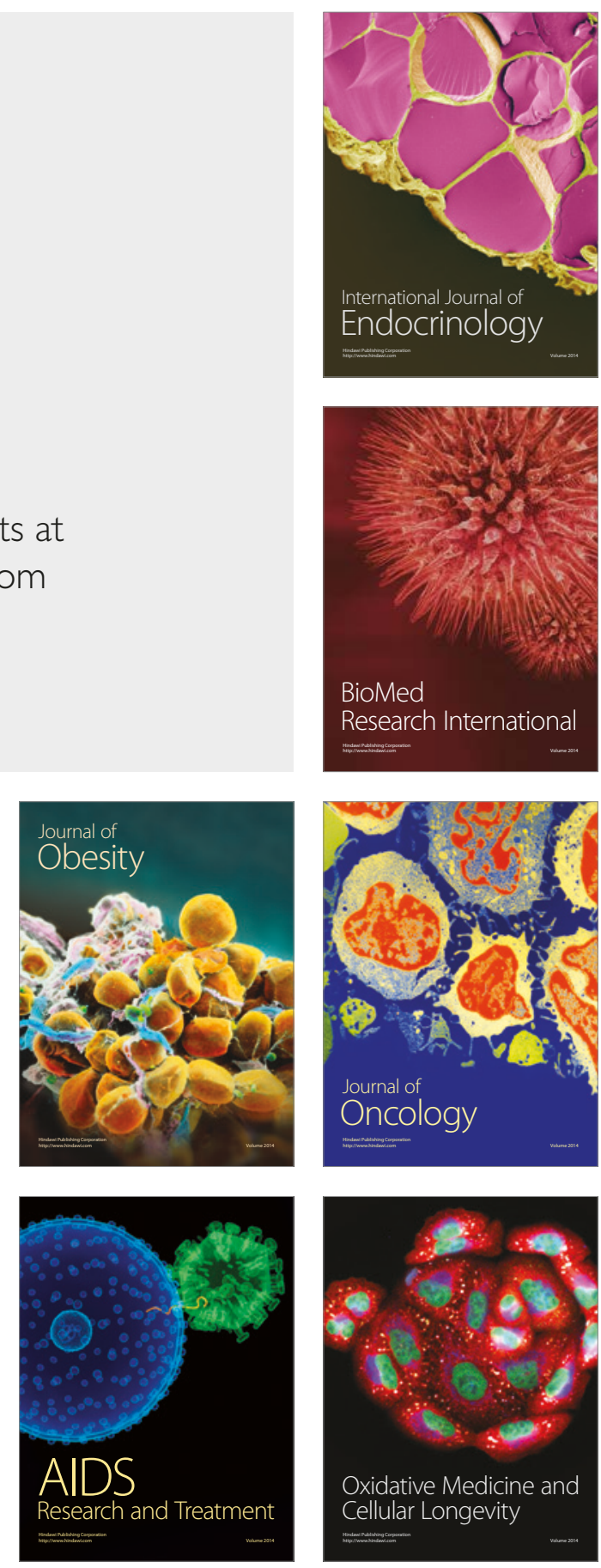